\title{
Antidepressants modulate glycine action in rat hippocampus
}

\author{
Hyun-Kyung Chang', Khae Hawn Kim', Ki-Woon Kang ${ }^{2}$, Yoo-Jin Kang ${ }^{2}$, Tae-Wook Kim³ ${ }^{3}$ Hun-Kyung Park ${ }^{4}$, Sung-Eun Kim 4 , \\ Chang-Ju Kim ${ }^{4, *}$
}

'Department of Urology, Gachon University Gil Medical Center, Gachon University School of Medicine, Incheon, Korea

${ }^{2}$ Department of Anesthesiology and Pain Medicine, College of Medicine, Kyung Hee University, Seoul, Korea

${ }^{3}$ Department of Community Sport and Recreation, College of Health Science, Jangan University, Hwasung, Korea

${ }^{4}$ Department of Physiology, College of Medicine, Kyung Hee University, Seoul, Korea

Antidepressants are drugs that relieve symptoms of depressive disorders. Fluoxetine, tianeptine, and milnacipran are different types of antidepressants, and they have widely been used for relieving of depression symptoms. In the present study, the effects of fluoxetine, tianeptine, and milnacipran on the glycine-induced ion current by nystatin-perforated patch clamp and on the amplitude of field potential in the hippocampal CA1 region by multichannel extracellular recording, MED64, system, were studied. In the present results, fluoxetine, tianeptine, and milnacipran reduced glycine-induced ion current in the hippocampal
CA1 neurons in nystatin-perforated patch clamp method. These drugs enhanced the amplitude of the field potential in the hippocampal CA1 region in MED64 system. These results suggest that antidepressants may increase neuronal activity by enhancing field potential through inhibition on glycine-induced ion current.

Keywords: Antidepressants, Glycine-induced ion current, Field potential, Hippocampus

\section{INTRODUCTION}

Antidepressants are drugs that relieve symptoms of depressive disorders. There are above 30 different kinds of antidepressants available today, and these drugs are classified into four major groups: the monoamine oxidase inhibitors, the tricyclic antidepressants, the selective serotonin reuptake inhibitors (SSRIs), and the others called as heterocyclic antidepressants. Among them, SSRIs are widely used for the treatment of depression.

Fluoxetine, tianeptine, and milnacipran are different types of antidepressants, and they have widely been used for relieving of depression symptoms. Fluoxetine is a classic SSRI, and has been shown to be highly effective with little side effects (Bymaster et al., 2002). Tianeptine belongs to the selective serotonin reuptake enhancer (SSRE) (Fattaccini et al., 1990). Milnacipran is a serotonin and norepinephrine (NE) reuptake inhibitor (SNRI) (Deli-
ni-Stula, 2000). These antidepressants are related with serotonin (5-hydroxytryptamine, 5-HT) theory. Fluoxetine selectively inhibits serotonin reuptake pump from serotonergic nerve terminals, resulting in increment of serotonin concentration in synaptic cleft (Schmidt et al., 1988). Tianeptine increases the level of 5-hydroxyindoleacetic acid in hippocampus and hypothalamus, enhancing 5-HT uptake and its metabolism (Marinesco et al., 1996). Milnacipran acts on the 5-HT and NE neurons, and shows long-term efficacy in patients with major depression (Mongeau et al., 1998). Chronic administration of milnacipran significantly increases basal synthesis of 5-HT and NE (Kamata et al., 2003).

The amino acid glycine is a major inhibitory neurotransmitter in the brain and spinal cord. Glycine has two physiological synaptic functions: it enhances the N-methyl-D-aspartate response at concentrations less than $1 \mu \mathrm{M}$, and at higher concentrations, it activates strychnine-sensitive glycine receptors (GLYRs), resulting in chlo-
${ }^{*}$ Corresponding author: Chang-Ju Kim

Department of Physiology, College of Medicine, Kyung Hee University,

26 Kyungheedae-ro, Dongdaemun-gu, Seoul 02447, Korea

Tel: +82-2-961-0407, Fax: +82-2-964-2195, E-mail: changju@kgu.ac.kr

Received: November 13, 2015 / Accepted: December 13, 2015
This is an Open Access article distributed under the terms of the Creative Commons Attribution Non-Commercial License (http://creativecommons.org/licenses/by-nc/3.0/) which permits unrestricted non-commercial use, distribution, and reproduction in any medium, provided the original work is properly cited. 
ride-mediated neuronal inhibition (Pourcho et al., 1992). GLYRs are widely distributed in the hippocampus besides spinal cord and brain stem (Chattipakorn and McMahon, 2002). Glycine synapses in the brain stem and spinal cord are implicated in the transmission of nociception, in which glycine inhibits neurotransmission and relieves pain. Moreover, some glycine transpoters are associated with neurotransmission by modulation of glycine concentration at the synaptic cleft in glycinergic neurons. Chronic treatment with antidepressants reduced the potency of glycine (Nowak et al., 1993).

Field potential is extracellular potential induced by membrane electromotive force. The field potential at any point in the medium is given by the sum of potentials resulting from each of the current sourced, weighed according to their distances and the distribution of conductivity in the medium (Zhou and Poon, 2000). Measuring of the field potential is a technique that has widely been used for the synaptic transmission in the hippocampus, neocortex, and other brain regions. Field potential in the hippocampal CA1 region represents field excitatory postsynaptic potential (fEPSP) (Zhou and Poon, 2000). Simultaneous recording of many sites in the brain enables the detection of brain networks in spatiotemporal patterns (Shimono et al., 2002).

The neurophysiological mechanisms underlying the antidepressants still remains unclear, and the exact mechanisms in relation with neuronal excitability and synaptic transmission in the hippocampus have not been clarified yet. In the present study, the effects of fluoxetine, tianeptine, and milnacipran on the glycine-induced ion current by nystatin-perforated patch clamp and on the amplitude of field potential in the hippocampal CA1 region by multichannel extracellular recording, MED64, system, were studied.

\section{MATERIALS AND METHODS}

\section{Patch clamp recording \\ Preparation of hippocampal CA1 neurons}

Hippocampal CA1 neurons were freshly dissociated using technique described previously elsewhere (Kim et al., 2002; Yang et al., 2003). In brief, 10- to 15-day-old Sprague-Dawley rats of both sexes were decapitated under Zoletil 50 anesthesia $(50 \mathrm{mg} /$ $\mathrm{kg}$; intramuscular).

The brain was removed and the transverse slices $(400-\mu \mathrm{m}$ thickness) were made with a microslicer (DTK-1000, DSK, Tokyo, Japan). Slices were preincubated in the incubation solution that had been well saturated with $95 \% \mathrm{O}_{2}$ and $5 \% \mathrm{CO}_{2}$ at room temperature for $30 \mathrm{~min}$. Then, the slices were treated with pronase (protease XIV, $1 \mathrm{mg} / 6 \mathrm{~mL}$ of the oxygenated incubation solution) for
$40-80 \mathrm{~min}$ at $32^{\circ} \mathrm{C}$ and subsequently with thermolysin (protease $\mathrm{X}, 1 \mathrm{mg} / 6 \mathrm{~mL}$ ) for $10-20 \mathrm{~min}$ at $32^{\circ} \mathrm{C}$. After enzyme treatment, the slices were kept in the enzyme free incubation solution for $1 \mathrm{hr}$.

The hippocampal CA1 region was identified in a $60-\mathrm{mm}$ culture dish coated with silicone under a binocular microscope (SZST, Olympus, Tokyo, Japan), and was micropunched out from the slices with an electrolytically polished injection needle. The micropunched hippocampal CA1 neurons were mechanically dissociated in a different dish with fire-polished fine glass Pasteur pipettes in 35-mm plastic culture dishes (3801, Falcon, Franklin Lakes, NJ, USA) filled with standard solution. The dissociation procedure was done under an inverted phase-contrast microscope (CK-2, Olympus, Tokyo, Japan). The dissociated neurons usually adhered to the bottom of the dish within $20 \mathrm{~min}$. These cells were remained viable for electrophysiological studies up to $6 \mathrm{hr}$ after dissociation.

\section{Electrical measurements}

Electrical recordings were performed in the nystatin-perforated patch recording mode under voltage-clamp condition (Kim et al., 2002; Yang et al., 2003). Patch pipette was prepared from glass capillaries with an outer diameter of $1.5 \mathrm{~mm}$ on a two-stage puller (PB-7, Narishige, Tokyo, Japan). The resistance between the recording electrode filled with the internal pipette solution and the reference electrode was 6-8 $8^{\mathrm{M} Q}$. After stable perforated patch formation, the series resistance ranged from 16 to $25^{\mathrm{MQ}}$.

Electrical stimulation, current recordings, and filtration of currents (at $2.9 \mathrm{kHz}$ ) were obtained with an EPC-7 patch-clamp amplifier (List-Electronic, Darmstadt/Eberstat, Germany). The current and voltage were monitored on a pen recorder (Recti-Horiz8K, NEC San-ei, Tokyo, Japan). All experiments were performed at room temperature $\left(22^{\circ} \mathrm{C}-24^{\circ} \mathrm{C}\right)$.

Drugs were added to the standard solution at the final concentrations provided in the text and were applied using a rapid application system termed the "Y-tube method" as described elsewhere (Kim et al., 2002; Yang et al., 2003). By this technique, the standard solution surrounding a neuron could be exchanged within 10-20 ms.

\section{MED64 system recording}

\section{Preparation of hippocampal slices}

The general procedure for the preparation of the hippocampal slices was same as previously described (Shimono et al., 2002; Yi et al., 2009). In brief, 3- to 4-week-old Sprague-Dawley rats of both sexes were sacrificed by decapitation after anesthesia using Zoletil 50 (50 mg/kg; intramuscular), and whole brain was removed carefully. The brain was immediately soaked in ice-cold ox- 
ygenated incubation solution within 1 min. Appropriate portions containing hippocampus of the brain were placed on the ice-cold plate of the vibrating tissue slicer (DTK-1000, DSK) and transverse slices (300- $\mu \mathrm{m}$ thickness) were made. Slices were incubated in the incubation solution which had been well saturated through exposure to $95 \% \mathrm{O}_{2}$ and $5 \% \mathrm{CO}_{2}$ for $1 \mathrm{hr}$ at room temperature.

\section{Electrical measurements}

Electrophysiological recording was measured by 64-channel multielectrode dish of the MED64 system (Alpha MED Science, Tokyo, Japan), a novel two-dimensional electroactivity monitoring technique described elsewhere (Shimono et al., 2002; Yi et al., 2009). A slice was placed on the center on the MED probe, which was treated with coating solution for $8 \mathrm{hr}$ at room temperature, and positioned to cover the $8 \times 8$ microelectrode arrays with 150 $\mu \mathrm{m}$ interpolar distance. After positioning of the slice on MED probe, microscopic photograph was taken. MED probe was placed on the MED connector and incubation solution oxygenated with $95 \% \mathrm{O}_{2}$ and $5 \% \mathrm{CO}_{2}$ was continuously infused at the rate of 2 $\mathrm{mL} / \mathrm{min}$ (at $34^{\circ} \mathrm{C}$ ). Evoked field potentials on 64 sites were simultaneously recorded after selecting of one recording site and two stimulation sites. For the recording of evoked field potentials, a pair of single planar microelectrodes with bipolar constant current pulses (45-90 $\mu \mathrm{A}, 0.1 \mathrm{~ms}$ ) was applied. Amplitude of field potential recorded in the absence of drug was used as a control value, and then amplitude of field potential recorded during drug application was measured.

\section{Solutions}

The ionic composition of the incubation solutions was (in $\mathrm{mM}$ ): $\mathrm{NaCl} 124, \mathrm{KCl} 5, \mathrm{KH}_{2} \mathrm{PO}_{4} 1.2, \mathrm{MgSO}_{4} 1.3, \mathrm{CaCl}_{2} 2.4$, glucose 10 , and $\mathrm{NaHCO}_{3} 24$. The $\mathrm{pH}$ was adjusted to $\mathrm{pH} 7.4$ by continuous bubbling with $95 \% \mathrm{O}_{2}$ and $5 \% \mathrm{CO}_{2}$ and was maintained at $34^{\circ} \mathrm{C}$ in water bath. The composition of the standard external solution was (in $\mathrm{mM}$ ): $\mathrm{NaCl} 150, \mathrm{KCl} 5, \mathrm{MgCl}_{2} 1, \mathrm{CaCl}_{2}$ 2, glucose 10, and $N$-2-hydroxyethylpiperazine- $N^{\prime}$-2-ethanesulphonic acid (HEPES) 10. The $\mathrm{pH}$ was adjusted to the 7.4 with tris-hydroxymethylaminomethane (Tris-base). The composition of the internal pipette solution for nystatin perforated recording contained (in $\mathrm{mM}$ ): $\mathrm{KCl} 150$ and HEPES 10. The $\mathrm{pH}$ was adjusted to 7.2 by adding Tris-base. A stock solution containing $10 \mathrm{mg} /$ $\mathrm{mL}$ nystatin in methanol was prepared and added in a final concentration of $200 \mu \mathrm{g} / \mathrm{mL}$ to the patch pipette solution. The composition of the probe coating solution contained $0.1 \%$ polyethylenimine in $25 \mathrm{mM}$ borate buffer adjusted $\mathrm{pH}$ 8.4.

\section{Drugs}

Zoletil 50 was obtained from Vibac Laboratories (Carros, France). Fluoxetine was purchased from Sigma Chemical Co. (St. Louis, MO, USA). Tianeptine was obtained from SERVIER (Courbevoie, France). Milnacipran was obtained from Cypress Bioscience Inc. (San Diego, CA, USA). Glycine, polyethylenimine, pronase, thermolysin, nystatin, and most of the other drugs used in this study were purchased from Sigma Chemical Co.

\section{Statistical analysis}

The data was analyzed was performed using one-way analysis of variance followed by Tukey multiple comparison tests. The results were expressed as the mean \pm standard error of the mean and $P$-value less than 0.05 was considered as a statistical significance.

\section{RESULTS}

\section{Effects of antidepressants on glycine-induced ion current in rat hippocampal CA1 neurons}

In the nystatin-perforated patch-clamp mode, experiments were
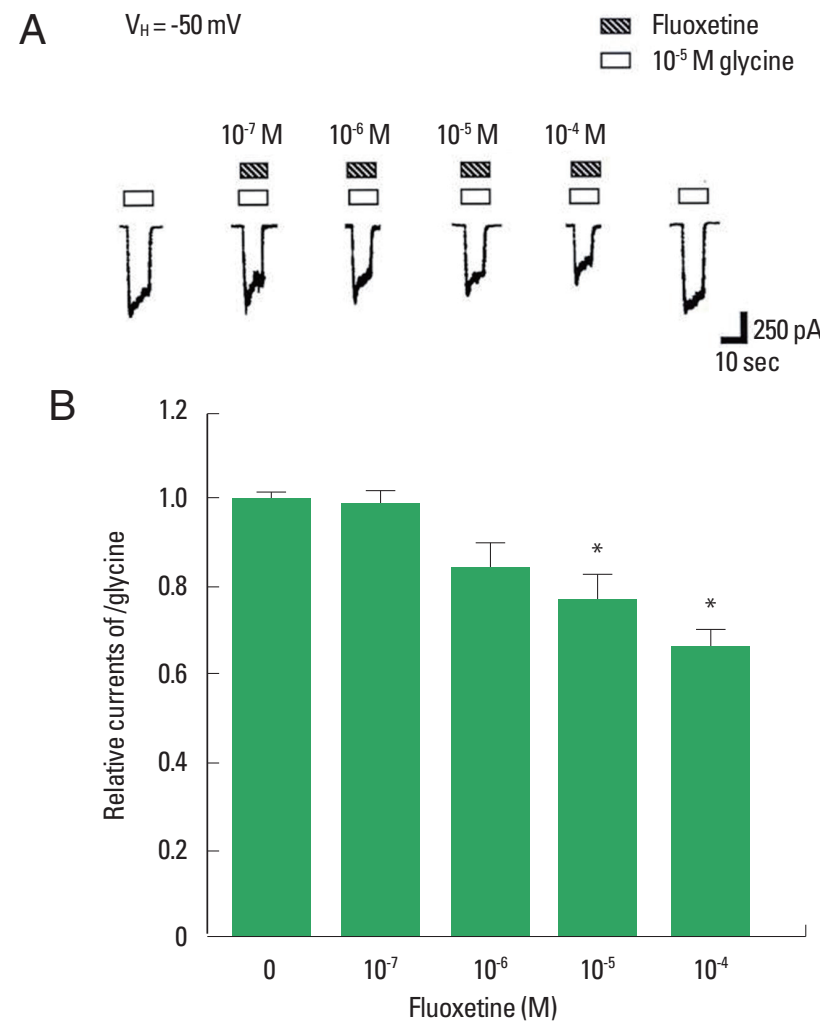

Fig. 1. Modulation of fluoxetine on glycine-activated ion current. Glycine-induced ion current was significantly inhibited by $10^{-5} \mathrm{M}$, and $10^{-4} \mathrm{M}$ of fluoxetine. ${ }^{*} P<0.05$ compared to the control. 
carried out at a holding potential $\left(\mathrm{V}_{\mathrm{H}}\right)$ of $-50 \mathrm{mV}$. To investigate the modulation of antidepressants on the glycine-induced ion current, the magnitude of ion current elicited by $10^{-5} \mathrm{M}$ glycine was used as the control value set as 1 . Antidepressants were applied every $2 \mathrm{~min}$ and ion current-activated by $10^{-5} \mathrm{M}$ glycine.

\section{Modulation of fluoxetine on glycine-induced ion current}

To investigate the modulation of fluoxetine on the glycine-induced ion current, the magnitude of ion current elicited by $10^{-5} \mathrm{M}$ glycine was used as the control value and $10^{-7} \mathrm{M}, 10^{-6} \mathrm{M}, 10^{-5} \mathrm{M}$, and $10^{-4} \mathrm{M}$ fluoxetine were applied simultaneously with $10^{-5} \mathrm{M}$ glycine. Fluoxetine at concentrations of $10^{-7} \mathrm{M}, 10^{-6} \mathrm{M}, 10^{-5} \mathrm{M}$, and $10^{-4} \mathrm{M}$ suppressed glycine-induced ion current to $0.99 \% \pm 0.03 \%$, $0.84 \% \pm 0.06 \%, 0.77 \% \pm 0.05 \%$, and $0.66 \% \pm 0.04 \%$, with the control value set as 1 . In the present study, the glycine-induced ion current in the hippocampal CA1 neurons was suppressed by application of fluoxetine (Fig. 1).

A<smiles>[Y3][As][13CH]</smiles>

Tianeptine $\square 10^{-5} \mathrm{M}$ glycine

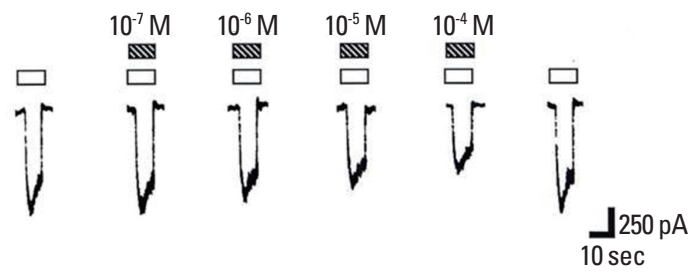

B

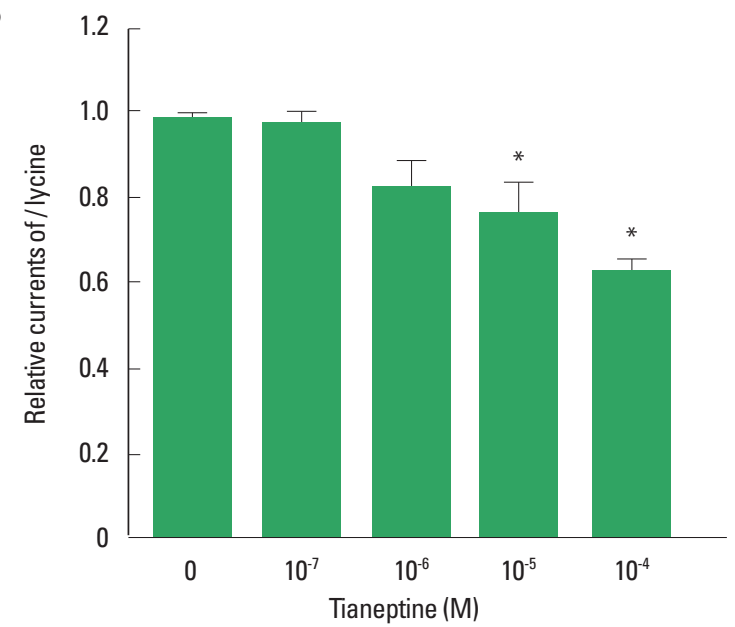

Fig. 2. Modulation of tianeptine on glycine-activated ion current. Glycine-induced ion current was significantly inhibited by $10^{-5} \mathrm{M}$, and $10^{-4} \mathrm{M}$ of tianeptine. ${ }^{*} P<0.05$ compared to the control.

\section{Modulation of tianeptine on glycine-induced ion current}

To investigate the modulation of tianeptine on the glycine-induced ion current, the magnitude of ion current elicited by $10^{-5} \mathrm{M}$ glycine was used as the control value and $10^{-7} \mathrm{M}, 10^{-6} \mathrm{M}, 10^{-5} \mathrm{M}$, and $10^{-4} \mathrm{M}$ tianeptine were applied simultaneously with $10^{-5} \mathrm{M}$ glycine. Tianeptine at concentrations of $10^{-7} \mathrm{M}, 10^{-6} \mathrm{M}, 10^{-5} \mathrm{M}$, and $10^{-4}$ M suppressed glycine-induced ion current about $0.99 \% \pm 0.03 \%$, $0.84 \% \pm 0.06 \%, 0.77 \% \pm 0.07 \%$, and $0.63 \% \pm 0.03 \%$ of the control value set as 1 . In the present study, the glycine-induced ion current in the hippocampal CA1 neurons was suppressed by application of tianeptine (Fig. 2).

\section{Modulation of milnacipran on glycine-induced ion current}

To investigate the modulation of milnacipran on the glycine-induced ion current, the magnitude of ion current elicited by $10^{-5} \mathrm{M}$ glycine was used as the control value and $10^{-7} \mathrm{M}, 10^{-6} \mathrm{M}, 10^{-5} \mathrm{M}$, and $10^{-4} \mathrm{M}$ milnacipran were applied simultaneously with $10^{-5} \mathrm{M}$ glycine. Milnacipran at concentrations of $10^{-7} \mathrm{M}, 10^{-6} \mathrm{M}, 10^{-5} \mathrm{M}$, and $10^{-4} \mathrm{M}$

A

$$
\begin{aligned}
V_{H}=-50 \mathrm{mV} & \text { Milnacipran } \\
& \square 10^{-5} \mathrm{M} \text { glycine }
\end{aligned}
$$

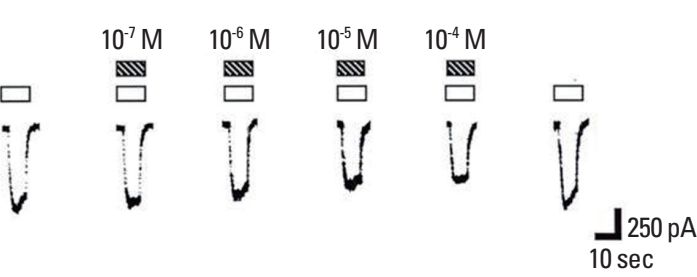

B

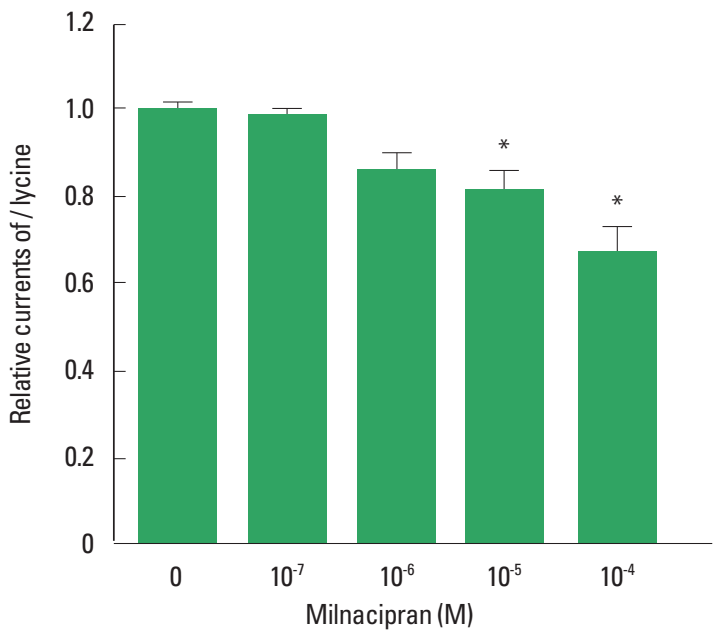

Fig. 3. Modulation of milnacipran on glycine-activated ion current. Glycine-induced ion current was significantly inhibited by $10^{-5} \mathrm{M}$, and $10^{-4} \mathrm{M}$ of milnacipran. ${ }^{*} P<0.05$ compared to the control. 
suppressed glycine-induced ion current about $0.99 \% \pm 0.08 \%$, $0.86 \% \pm 0.03 \%, 0.81 \% \pm 0.04 \%$, and $0.67 \% \pm 0.05 \%$ of the control value set as 1 . In the present study, the glycine-induced ion current in the hippocampal CA1 neurons was suppressed by application of milnacipran (Fig. 3).

\section{Effect of glycine on antidepressants-induced field potential in rat hippocampal slices}

Experiment was carried out by applying a fixed pulse of 45-90 $\mu \mathrm{V}$. Amplitude of the field potential in the CA1 region was recorded without drug applications for $10 \mathrm{~min}$, and used as a control value. Each antidepressant was applied at the rate of $2 \mathrm{~mL} /$ min for 10 min following measuring of control value. The amplitude change induced by antidepressants was expressed as a percent (\%) of the control value set as $100 \%$.

\section{Effect of glycine on fluoxetine-induced field potential}

In order to evaluate the involvement of the inhibitory neurotrans- mitter glycine on fluoxetine-activated field potential in rat hippocampal slices, $10^{-5} \mathrm{M}$ of glycine was coapplied on the hippocampal slices with $10^{-5} \mathrm{M}$ of fluoxetine and amplitude of the field potential was recorded for $20 \mathrm{~min}$. The amplitude of field potential in the CA1 region was similarly continued to $100.58 \% \pm 2.93 \%$ of the control value set as $100 \%$ by treatment with $10^{-5} \mathrm{M}$ of glycine and $10^{-5} \mathrm{M}$ of fluoxetine. Subsequent treatment with $10^{-5} \mathrm{M}$ fluoxetine in the absence of glycine for $20 \mathrm{~min}$ enhanced the field potential to $135.50 \% \pm 6.20 \%$ of the control value. After maximum increase of field potential by fluoxetine treatment, hippocampal slices was washed with incubation solution for $40 \mathrm{~min}$. The field potential enhanced by fluoxetine application was diminished to $114.50 \% \pm$ $8.20 \%$ of the control value by wash out (Fig. 4).

In the present study, coapplication of glycine and fluoxetine inhibited the field potential to the control value, but treatment with only fluoxetine was shown to enhance field potential amplitude in the CA1 region and of hippocampus. The increased field potential by fluoxetine application was recovered as control value by wash out.
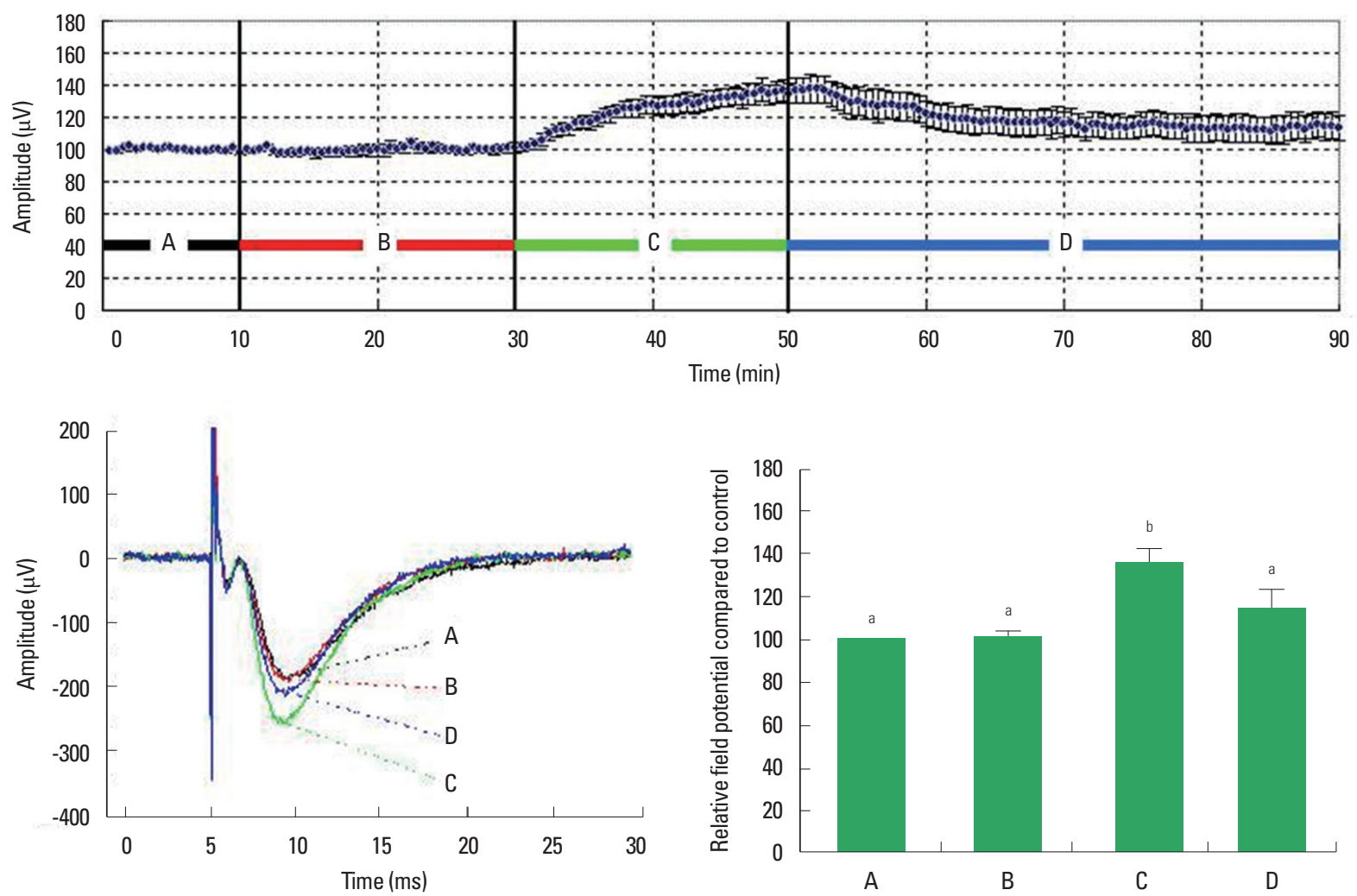

Fig. 4. Effect of glycine on fluoxetine-induced field potential amplitude in rat hippocampal slices. Above: Dot plot of relative field potential amplitude with time. Each dot was recoded as 30-sec intervals. Left below: Schematic representation of field potential. Right below: Relative field potential compared to control. Letters (a, b) mean statistical significance $P<0.05$. (A) Control group in the absence of any drugs, (B) $10^{-5} \mathrm{M}$ of glycine and $10^{-5} \mathrm{M}$ fluoxetine-treated group, (C) $10^{-5} \mathrm{M}$ fluoxetine-treated group, (D) washing group with incubation solution. 

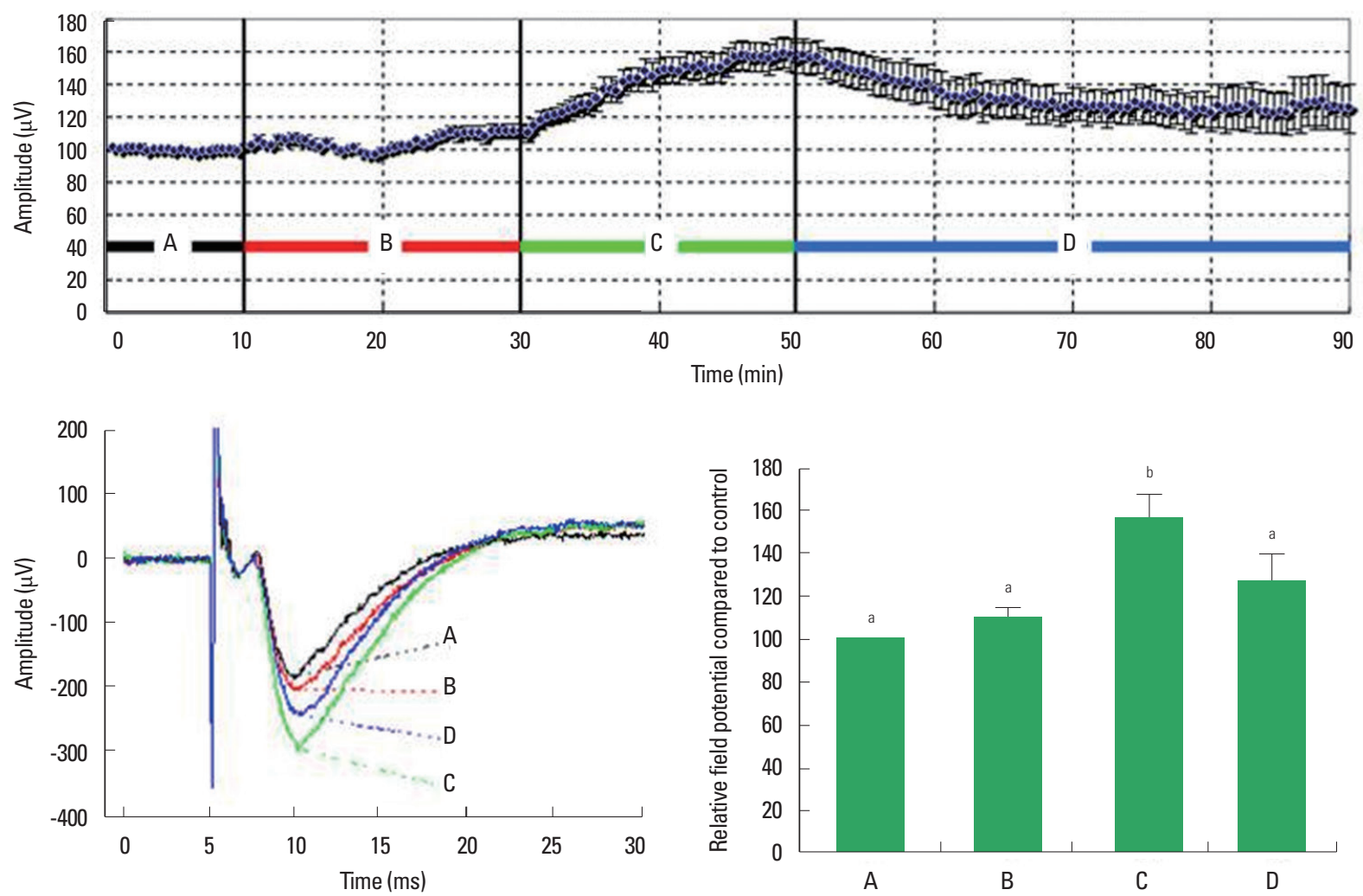

Fig. 5. Effect of glycine on tianeptine-induced field potential amplitude in rat hippocampal slices. Above: Dot plot of relative field potential amplitude with time. Each dot was recoded as 30-sec intervals. Left below: Schematic representation of field potential. Right below: Relative field potential compared to control. Letters (a, b) mean statistical significance $P<0.05$. (A) Control group in the absence of any drugs, (B) $10^{-5} \mathrm{M}$ of glycine and $10^{-5} \mathrm{M}$ tianeptine-treated group, (C) $10^{-5} \mathrm{M}$ tianeptine-treated group, (D) washing group with incubation solution.

\section{Effect of glycine on tianeptine-induced field potential}

In order to evaluate the involvement of the inhibitory neurotransmitter glycine on tianeptine-activated field potential in rat hippocampal slices, $10^{-5} \mathrm{M}$ of glycine was coapplied on the hippocampal slices with $10^{-5} \mathrm{M}$ of tianeptine and amplitude of the field potential was recorded for $20 \mathrm{~min}$. The amplitude of field potential in the CA1 region was similarly continued to $110.98 \% \pm 4.25 \%$ of the control value set as $100 \%$ by treatment with $10^{-5} \mathrm{M}$ of glycine and $10^{-5} \mathrm{M}$ of tianeptine. Subsequent treatment with $10^{-5} \mathrm{M}$ tianeptine in the absence of glycine for 20 min enhanced the field potential to $157.76 \% \pm 9.41 \%$ of the control value. After maximum increase of field potential by tianeptine treatment, hippocampal slices was washed with incubation solution for $40 \mathrm{~min}$. The field potential enhanced by tianeptine application was diminished to $125.98 \% \pm 14.07 \%$ of the control value by wash out (Fig. 5).

In the present study, coapplication of glycine and tianeptine inhibited the field potential to the control value, but treatment with only tianeptine was shown to enhance field potential amplitude in the CA1 region and of hippocampus. The increased field potential by tianeptine application was partially recovered to control value by washout.

\section{Effect of glycine on milnacipran-induced ion current}

In order to evaluate the involvement of the inhibitory neurotransmitter glycine on milnacipran-activated field potential in rat hippocampal slices, $10^{-5} \mathrm{M}$ of glycine was coapplied on the hippocampal slices with $10^{-5} \mathrm{M}$ of milnacipran and amplitude of the field potential was recorded for $20 \mathrm{~min}$. The amplitude of field potential in the CA1 region was similarly continued to $101.10 \% \pm 3.44 \%$ of the control value set as $100 \%$ by treatment with $10^{-5} \mathrm{M}$ of glycine and $10^{-5} \mathrm{M}$ of milnacipran. Subsequently treatment with $10^{-5} \mathrm{M}$ milnacipran in the absence of glycine for 20 min enhanced the field potential to $147.14 \% \pm 7.60 \%$ of the control value. After maximum increase of field potential by milnacipran treatment, hippocampal slices was washed with incubation solution for $40 \mathrm{~min}$. The field potential enhanced by milnacipran application was diminished to $106.49 \% \pm 5.02 \%$ of the control value by wash out (Fig. 6). 

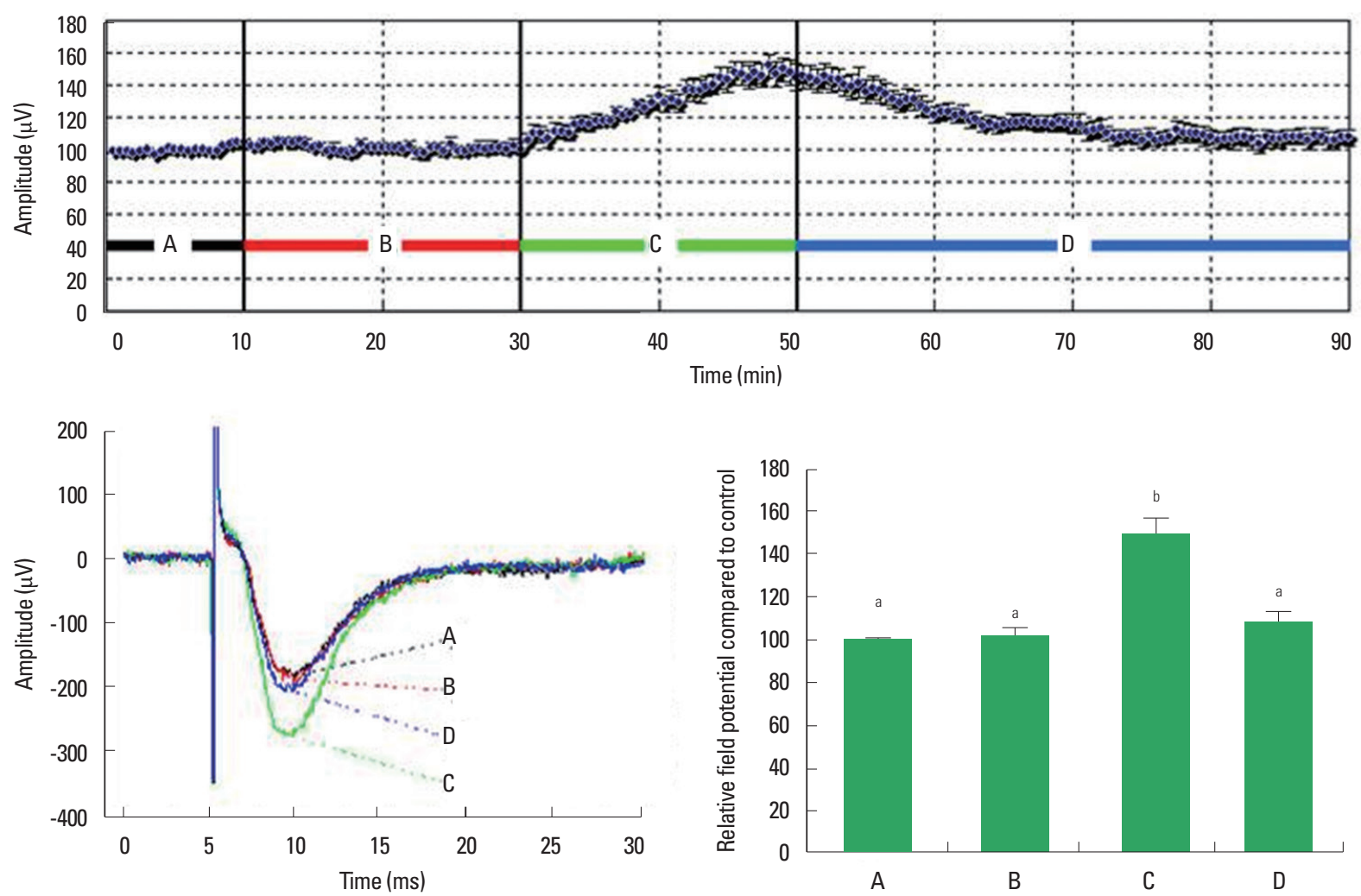

Fig. 6. Effect of glycine on milnacipran-induced field potential amplitude in rat hippocampal slices. Above: Dot plot of relative field potential amplitude with time. Each dot was recoded as 30-sec intervals. Left below: Schematic representation of field potential. Right below: Relative field potential compared to control. Letters (a, b) mean statistical significance $P<0.05$. (A) Control group in the absence of any drugs, (B) $10^{-5} \mathrm{M}$ of glycine and $10^{-5} \mathrm{M}$ milnacipran-treated groupa, (C) $10^{-5} \mathrm{M}$ milnacipran-treated group, (D) washing group with incubation solution.

In the present study, coapplication of glycine and milnacipran inhibited the field potential to the control value, but treatment with only milnacipran was shown to enhance field potential amplitude in the CA1 region and of hippocampus. The increased field potential by milnacipran application was recovered as control value by wash out.

\section{DISCUSSION}

In the present study, we investigated the modulation of antidepressants on the glycine-induced ion current. The results showed that fluoxetine, tianeptine, and milnacipran reduced glycine-induced ion current in the hippocampal CA1 neurons (Figs. 1-3).

Glycine application to GLYRs expressing neurons in acutely dissociated hippocampal slices in rats induced strychnine-sensitive currents which suppress neuronal excitability (Chattipakorn and McMahon 2002). GLYRs are expressed throughout the hippocampus, and they are characterized as glycine-gated chloride current in rat hippocampal slices pharmacologically (Brackmann et al., 2004; Mori et al., 2002). Binding of glycine to its receptors produces a large increase in chloride conductance, which causes membrane hyperpolarization (Chen et al., 1996).

In the present study, fluoxetine, tianeptine, and milnacipran enhanced field potential in the hippocampal CA1 region, independently to the types of these antidepressants such as SSRI, SSRE, and SNRI (Figs. 4-6). Moreover the magnitudes of field potential amplitude were maintained control level by coapplication of glycine and antidepressants. These results suggest that antidepressants inhibited glycine action, thus enhanced field potential in the hippocampal CA1 region. However extrasupplementation of glycine overcame the inhibitory action of antidepressants, thus field potential amplitude was maintained at control level. Here in this study, we have shown that antidepressants have inhibitory effect on glycine action.

Field potential represents the net balance of all currents that flow into a small, local volume in the extracellular fluid (Zhou and Poon, 2000). Release of excitatory neurotransmitters causes a local change in membrane potential, named excitatory postsynaptic po- 
tential (EPSP). EPSP generates a potential gradient along the membrane that, in turn, gives rise to the field potential, which reflects activities in hundreds to thousands of neurons in the brain.

In some studies, antidepressants, for example trimipramine, were reported not to increase the population and amplitude of spike potential in the hippocampus or eventually reduced the population and amplitude (Langosch and Walden, 2002). In other studies, however, it was reported that fEPSP in the hippocampus was increased by antidepressants. The repeated administration of imipramine enhanced adenosine-induced fEPSP (Zahorodna and Bijak, 1999).

In the present results, fluoxetine, tianeptine, and milnacipran reduced glycine-induced ion current in the hippocampal CA1 neurons in nystatin-perforated patch clamp method. Fluoxetine, tianeptine, and milnacipran enhanced the amplitude of the field potential in the hippocampal CA1 region in multichannel extracellular recording MED64 system. The magnitudes of field potential amplitude were maintained control level by coapplication of glycine and antidepressants. These results show that antidepressants can increase neuronal activity by enhancing field potential through inhibition on glycine-induced ion current.

\section{CONFLICT OF INTEREST}

No potential conflict of interest relevant to this article was reported.

\section{ACKNOWLEDGMENTS}

This work was supported by the National Research Foundation of Korea Grant funded by the Korean Government (NRF-20100022895).

\section{REFERENCES}

Brackmann M, Zhao C, Schmieden V, Braunewell KH. Cellular and subcellular localization of the inhibitory glycine receptor in hippocampal neurons. Biochem Biophys Res Commun 2004;324:1137-1142.

Bymaster FP, Zhang W, Carter PA, Shaw J, Chernet E, Phebus L, Wong DT, Perry KW. Fluoxetine, but not other selective serotonin uptake inhibitors, increases norepinephrine and dopamine extracellular levels in prefrontal cortex. Psychopharmacology (Berl) 2002;160:353-361.

Chattipakorn SC, McMahon LL. Pharmacological characterization of glycine-gated chloride currents recorded in rat hippocampal slices. J Neurophysiol 2002;87:1515-1525.

Chen G, Trombley PQ, van den Pol AN. Excitatory actions of GABA in developing rat hypothalamic neurones. J Physiol 1996;494(Pt 2):451464.

Delini-Stula A. Milnacipran: an antidepressant with dual selectivity of action on noradrenaline and serotonin uptake. Hum Psychopharmacol 2000;15:255-260.

Fattaccini CM, Bolaños-Jimenez F, Gozlan H, Hamon M. Tianeptine stimulates uptake of 5-hydroxytryptamine in vivo in the rat brain. Neuropharmacology 1990;29:1-8.

Kamata M, Naito S, Takahashi H, Higuchi H. Milnacipran for the treatment of chronic pain. Hum Psychopharmacol 2003;18:575-576.

Kim YJ, Shin MC, Kim SA, Chung JH, Kim EH, Kim CJ. Modulation of tianeptine on ion currents induced by inhibitory neurotransmitters in acutely dissociated dorsal raphe neurons of Sprague-Dawley rats. Eur Neuropsychopharmacol 2002;12:417-425.

Langosch JM, Walden J. Effects of the atypical antidepressant trimipramine on neuronal excitability and long-term potentiation in guinea pig hippocampal slices. Prog Neuropsychopharmacol Biol Psychiatry 2002;26:299-302.

Marinesco S, Poncet L, Debilly G, Jouvet M, Cespuglio R. Effects of tianeptine, sertraline and clomipramine on brain serotonin metabolism: a voltammetric approach in the rat. Brain Res 1996;736:82-90.

Mongeau R, Weiss M, de Montigny C, Blier P. Effect of acute, short- and long-term milnacipran administration on rat locus coeruleus noradrenergic and dorsal raphe serotonergic neurons. Neuropharmacology 1998;37:905-918.

Mori M, Gähwiler BH, Gerber U. Beta-alanine and taurine as endogenous agonists at glycine receptors in rat hippocampus in vitro. J Physiol 2002;539(Pt 1):191-200.

Nowak G, Trullas R, Layer RT, Skolnick P, Paul IA. Adaptive changes in the N-methyl-D-aspartate receptor complex after chronic treatment with imipramine and 1-aminocyclopropanecarboxylic acid. J Pharmacol Exp Ther 1993;265:1380-1386.

Pourcho RG, Goebel DJ, Jojich L, Hazlett JC. Immunocytochemical evidence for the involvement of glycine in sensory centers of the rat brain. Neuroscience 1992;46:643-656.

Schmidt MJ, Fuller RW, Wong DT. Fluoxetine, a highly selective serotonin reuptake inhibitor: a review of preclinical studies. Br J Psychiatry Suppl 1988;(3):40-46.

Shimono K, Baudry M, Panchenko V, Taketani M. Chronic multichannel recordings from organotypic hippocampal slice cultures: protection from excitotoxic effects of NMDA by non-competitive NMDA antagonists. J Neurosci Methods 2002;120:193-202.

Yang HJ, Shin MC, Chang HK, Jang MH, Lee TH, Kim YJ, Chung JH, Kim CJ. Bupivacaine and ropivacaine suppress glycine- and glutamate-induced ion currents in acutely dissociated rat hippocampal 
neurons. Neurosci Lett 2003;344:33-36.

Yi JW, Lee BJ, Kim DO, Park SW, Choi YK, Chang HK, Kim CJ, Park JH. Effects of bupivacaine and ropivacaine on field potential in rat hippocampal slices. Br J Anaesth 2009;102:673-679.

Zahorodna A, Bijak M. Imipramine-induced increase in the inhibitory ef- fect of adenosine receptor activation in the hippocampus. Pol J Pharmacol 1999;51:423-428.

Zhou Z, Poon CS. Field potential analysis of synaptic transmission in spiking neurons in a sparse and irregular neuronal structure in vitro. J Neurosci Methods 2000;94:193-203. 